\title{
Transgenic Improvement of Photosynthetic Property in Tobacco Using E12 $\Omega$ Promoter System to Express Higher Level of Mouse Carbonic Anhydrase Activity in Cytoplasm
}

\author{
Kenji JINUSHI*, Keiichiro OKABE**1), Ken ISHIMARU***, Tatsuro HIROSE***, \\ Ryu OHSUGI***, Ryuichi ISHII* \\ *Graduate School of Agricultural and Life Sciences, University of Tokyo, Yayoi, Bunkyo-ku, Tokyo \\ 113-8657, Japan \\ **Institute of Cell Engineering, DDS R\&D Center, ADVANCE Co.Ltd., Ohhashi 2-8-18, Meguro, Tokyo \\ 153-0044, Japan \\ ***National Institute of Agrobiological Resources, Kannondai, Tsukuba, Ibaraki 305-0856, Japan \\ ${ }^{1)}$ Corresponding author E-mail:
}

Received 20 January 1999; accepted 16 August 1999

\begin{abstract}
Genetic challenge to express higher carbonic anhydrase (CA) activity in the cytoplasm of tobacco mesophyll cells was carried out by an improved promoter (El2 $\Omega$ ) with a cDNA of partially modified mouse CA. In leaves of $\mathrm{T} 1$ progenies of transgenic tobacco (ffCA plant), the expressed mouse CA peptide was confirmed with $1.5-3.2$ times higher than the transgenic tobacco having foreign mouse CA controlled by simple CaMV 35S promoter (fCA plant). The ffCA plants manifested higher photosynthetic carbon assimilation (PCA) rate under ambient $\mathrm{CO}_{2}$ condition than wild type plants. The chlorophyll fluorescence measurement analysis revealed that the ffCA plant's improved PCA rate is related to the reduction of mesophyll $\mathrm{CO}_{2}$ transfer resistance (Rr) with little change in the stomatal resistance (Rs) or mesophyll $\mathrm{CO}_{2}$ fixation resistance (Rx). Moreover, ffCA plants showed a higher growth rate under high light condition.
\end{abstract}

\section{Abbreviations}

CA: carbonic anhydrase, CaMV: cauliflower mosaic virus, ffCA plants: the transgenic tobacco having foreign mouse CA controlled by El $\Omega$ promoter, fCA plants: the transgenic tobacco having foreign mouse CA controlled by simple CaMV $35 \mathrm{~S}$ promoter, PCA: photosynthetic carbon assimilation, $\mathrm{Rr}$ : mesophyll $\mathrm{CO}_{2}$ transfer resistance, Rs: stomatal resistance, Rx: mesophyll $\mathrm{CO}_{2}$ fixation resistance, WA unit: Wilbur-Anderson CA activity unit

\section{Introduction}

Plant biotechnology in photosynthetic improvement is based on finding the limiting steps in the metabolic pathway in the plant's cellular physiology. Carbonic anhydrase [EC4.2.1.1, CA], which catalyzes the conversion reaction between $\mathrm{CO}_{2}$ and $\mathrm{HCO}_{3}{ }^{-}$and also promotes the inorganic carbon diffusion through lipid membranes (Broun et al., 1970), is highly related to the flow of carbon in the leaf photosynthesis. Although there have been some reports suggesting the significant existence of extra- chloroplastic CA isozymes in several C3 species (Utsunomiya and Muto, 1993; Rumeau, 1996), most CA activity characteristically localizes in the chloroplastic stroma and not in its envelope (Tsuzuki $e t$ al., 1985). In the previous report (Jinushi et al., 1998), based on a very low level of cytoplasmic intrinsic CA activity we generated transgenic tobacco (fCA plants) which expresses foreign mouse CA in the mesophyll cytoplasm controlled by a conventional cauliflower mosaic virus $35 \mathrm{~S}$ (CaMV 35S) promoter. The fCA plants which expressed foreign $\mathrm{CA}$ at high levels showed a significant and consistent enhancing tendency in the photosynthetic carbon assimilation (PCA) rate under lower $\mathrm{CO}_{2}$ conditions. However, the strain that expressed foreign $\mathrm{CA}$ at a lower level did not show such an enhancement. These facts lead to hypothesis that the introduction of higher foreign CA activity into the cytoplasm would enhance its PCA activity to a greater extent.

Among the unique techniques available two reports were suggestive; (1) Kay et al. (1987) reported that the duplication of CaMV35S promoter sequences enhanced foreign gene expressions. (2) 
Mitsuhara et al. (1996) constructed a new CaMV35S promoter (E12 $\Omega$ ) containing two extraenhancer sequences; one is E12 sequence which is in the upstream region of CaMV35S promoter, and the other is $\Omega$ sequence which is the $5^{\prime}$-untranslated region of tobacco mosaic virus genomic RNA. They were observed to have more than 10 times higher expression of the marker gene using El $\Omega$ promoter compared with a single CaMV35S promoter in the transgenic tobacco.

In this paper we describe a study using El $2 \Omega$ promoter in the production of unique transgenic tobacco plants (ffCA), into which have been introduced a partially modified mouse CA cDNA and which expressed mouse CA peptide in the mesophyll cytoplasm. We measured those mesophyll resistances for $\mathrm{CO}_{2}$ transfer ( $\mathrm{Rr}$ ) in photosynthesis with chlorophyll fluorescence methods in order to elucidate the detailed mechanisms of introduced foreign CA on PCA.

\section{Materials and Methods}

\subsection{Construction of gene cassette with improved promoter and transformation}

The vector plasmid $\mathrm{pBffCA010}$ was constructed by replacing the sequence of $\beta$-glucuronidase gene and nos-terminator of the pBE2113-GUS (Mitsuhara et al., 1996) with the sequence of the partially modified mouse CA cDNA and nos-terminator in the pBfCA010 (Jinushi et al., 1998) of 861 bp at Bam HI -EcoRI site (Fig.1). It was introduced into leaf discs of tobacco (Nicotiana tabacum line SR-1) according to the same method as previously reported (Jinushi et al., 1998; Horsch et al., 1985). Transgenic tobacco plants were screened by antibiotic $\mathrm{Km}$ resistance and the expression level of foreign CA peptide using anti-serum against bovine CA type II which could discriminate it from plant intrinsic CA peptides.

[NPT-II gene cassette]

\subsection{Plant Materials}

T1 seeds of the transgenic tobacco plants, the strain ffCA-3, which showed an extremely high level of foreign CA expression, were sown on sand. The seedlings were grown in the growth cabinet where the temperature was maintained at $25^{\circ} \mathrm{C} / 20^{\circ} \mathrm{C}$ (day/night) with a 12 hour day length. The light intensity was kept constant at $1000 \mu \mathrm{mol} \cdot \mathrm{m}^{-2} \cdot \mathrm{s}^{-1}$ during day time. Two sets of the plants were prepared; one for the measurement of leaf intrinsic $\mathrm{CA}$ and foreign CA activity, and the other for the measurement of leaf PCA properties.

\subsection{Determination of CA Activity}

In the leaves of line SR - 1, which originated from the plastid genome mutation (Svab and Maliga, 1991), there were extremely low levels of endogenous CA activity compared to those of c.v. Xanthi, which we had previously tested to generate fCA transgenic plants (Jinushi et al., 1998). Fifteen $\mathrm{cm}^{2}$ of the leaves was ground with the mortar and pestle for $2 \mathrm{~min}$ on ice with $4 \%$ of polyvinyl polypyrrolidon, sea-sand and $1.4 \mathrm{ml}$ of the extraction buffer ( $\mathrm{pH} \mathrm{8.3)} \mathrm{containing} 50 \mathrm{mM}$ sodium barbital, $1 \mathrm{mM} \mathrm{Na} \mathrm{N}_{2}$-EDTA, $2 \%$ glycine, $2 \%$ polyvinyl pyrrolidon $1 \%$ BSA, $10 \%$ glycerol and $0.2 \mathrm{mM}$ phenylmethanesulfonylfluoride. After centrifugation at $4{ }^{\circ} \mathrm{C}$ and $15,000 \times \mathrm{g}$ for $5 \mathrm{~min}$, an aliquot of the supernatant was used for the measurement of CA activity (Tsuzuki et al., 1985). The foreign CA activity in the leaf extract was estimated by differential measurement in the presence and absence of $10^{-7} \mathrm{M}$ acetazolamide which inhibited the animal CA type II by $99 \%$ without any effect on leaf endogenous CA (Jinushi et al., 1998).

\subsection{Gas Exchange Measurement}

The PCA rates and fluorescence signals were simultaneously determined in the youngest fully

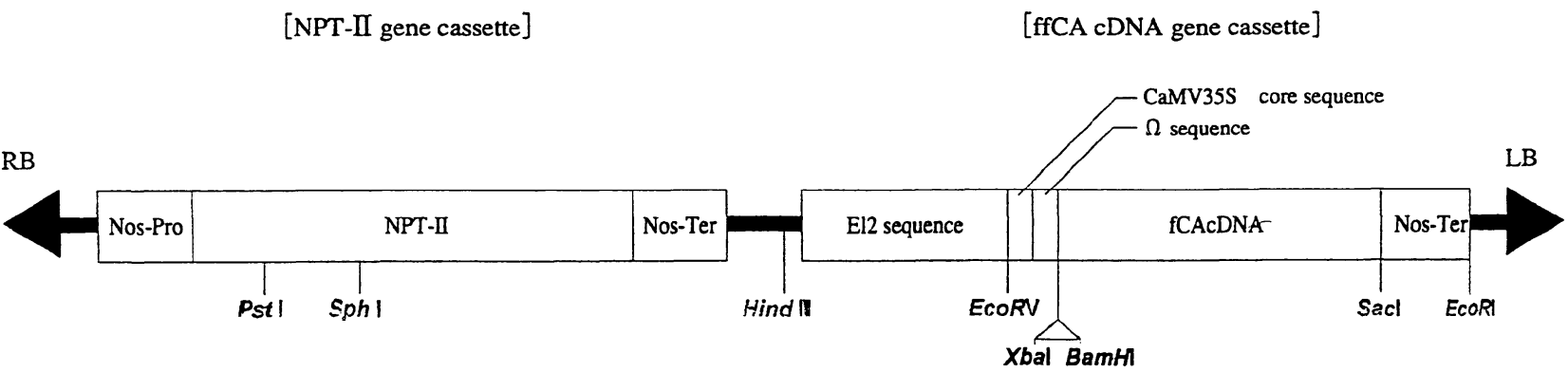

$1 \mathrm{~kb}$

Fig.1 The vector construction of pBffCA010; the ffCA cDNA gene cassette which was designed to express the partially modified mouse CA type II protein with total size of 252 amino acids at the left border and the NPTII gene cassette for the screening of transgenic plants at the right border. 
expanded leaves. In order to confirm the unique effects of cytoplasmic foreign $\mathrm{CA}$ activity specifically, sample plants with similar leaf conditions were selected by leaf chlorophyll content measured by a non-destructive method. The measuring system for the PCA rate was the same as reported in the previous paper (Jinushi et al., 1998; Nobel et al., 1975). The ambient $\mathrm{CO}_{2}$ concentration was sequentially changed in the order of 350,200, 100 and 80 $\mathrm{ppm}$. The light intensity, and the leaf temperature were maintained constant at $1000 \mu \mathrm{E} \cdot \mathrm{m}^{-2} \cdot \mathrm{s}^{-1}$ and $26 \pm 1{ }^{\circ} \mathrm{C}$, respectively. In some measurements, PCA was also determined under $350 \mathrm{ppm}$ of $\mathrm{CO}_{2}$ at $500 \mu \mathrm{E} \cdot \mathrm{m}^{-2} \cdot \mathrm{s}^{-1}$ of the light intensity.

\subsection{Chlorophyll Fluorescence Analysis}

To estimate $\mathrm{Rr}$, chlorophyll fluorescence parameters were measured with a PAM 101 modulated fluorometer (Waltz) (Genty et al., 1989; Harley et al., 1992; Schreiber, 1986). To measure the fluorescence signals simultaneously with PCA determination, a polyfurcated optic fiber was supported at angle of $45^{\circ}$ to the leaf chamber to avoid the shading of the leaf light illuminating.

The measurement of $\mathrm{Rr}$ value is known to be rather stable under lower $\mathrm{Ci}$ conditions (Loreto et al., 1992), thus, we compared the $\mathrm{Rr}$ values at $\mathrm{Ci}=$ $100 \mathrm{ppm}$. The calculated $\mathrm{Rr}$ value of Wild types of tobacco was nearly the same level as those reported in other papers which measured using the isotopic carbon method (Evans et al., 1986; Evans et al., 1994; von Caemmerer and Evans, 1991).

\subsection{Western Blot Analysis}

The RubisCO and expressed foreign CA levels in the leaves were measured by SDS-PAGE and Western blot analysis as described in the previous paper (Jinushi et al., 1998).

\subsection{Growth Analysis}

Using non-destructive leaf area measurement with a video camera and 2 -dimension pattern analysis program (ATTO, Japan), we selected transgenic and wild type seedlings of similar size at 5 weeks after sowing. They were cultured for 17 or 30 days under natural light or $50 \%$ shaded conditions in the green house. The temperature was maintained at $25^{\circ} \mathrm{C} / 20^{\circ} \mathrm{C}$ (day/night). Day time light intensity was at least $1000 \mu \mathrm{E} \cdot \mathrm{m}^{-2} \cdot \mathrm{s}^{-1}$ and the $\mathrm{CO}_{2}$ concentration was around $390 \mathrm{ppm}$.

\section{Results and Discussion}

The transgenic plants which showed a high level of foreign CA protein were confirmed by Western blot analysis of the leaf extracts of individuals of 4 strains (ffCA $-1,-3,-6$ and -7 ) among the 14 seedlings recovered from the screening antibiotic $\mathrm{Km}$ medium. By the PCR Southern and Northern analyses, it was confirmed that the mouse ffCA cDNA gene cassette existed in the chromosomal genome and was transcribed to messenger RNA in these 4 strains. However, two plantlets of these strains showed a strange appearance; the strain ffCA - 6 was dwarf with slender leaves, probably due to somaclonal variation and the strain ffCA-7 was sterile. Therefore, $\mathrm{T} 1$ seeds were obtained only from two remaining strains (ffCA-1 and ffCA-3). The segregation ratios of foreign CA expressing and non-expressing phenotypes in T1 plants were 27:0 (ffCA-1) and 33:4 (ffCA-3), which indicated that at least two copies of mouse CA cDNA gene cassette were introduced into the chromosomal DNA in these transgenic plants. Fig. 2 shows the Western blot analysis of leaf extracts in ffCA plants (ffCA-3) under the control of El $\Omega$ promoter and of fCA plants under the control of single CaMV35S promoter at 6 weeks after sowing. The expression level of foreign CA peptide in ffCA-3 was calculated with the 2 -dimentional-density pattern analysis program to be 3.2 times higher than the highest expression among the 23 fCA plants (High CA Plant). This indicated that the El $2 \Omega$ promoter could enhance the mouse CA cDNA gene expression just like GUS gene reported before (Mitsuhara et al., 1996). On the other hand, ffCA-1 showed a lower

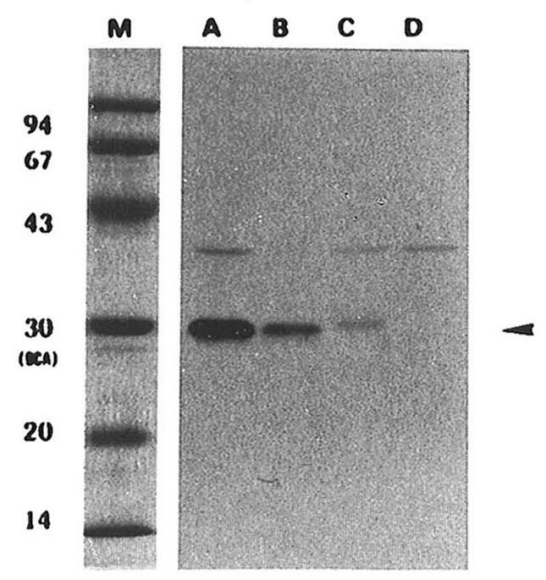

Fig. 2 Comparison of mouse CA expression level between transgenic plants containing different promoters. Lane $\mathrm{M}$ shows the molecular weight markers (Pharmacia) with $\mathrm{CBB}$ staining. The lanes $\mathrm{A}, \mathrm{B}, \mathrm{C}$, and $\mathrm{D}$, show the immunodetection of the leaf extract in the ffCA transgenic plant (the strain ffCA-3), in the High CA Plant and Low CA Plant of the fCA transgenic plant and in Wild Type, respectively. The arrow indicates the immuno-detection band of about $29 \mathrm{KD}$. 


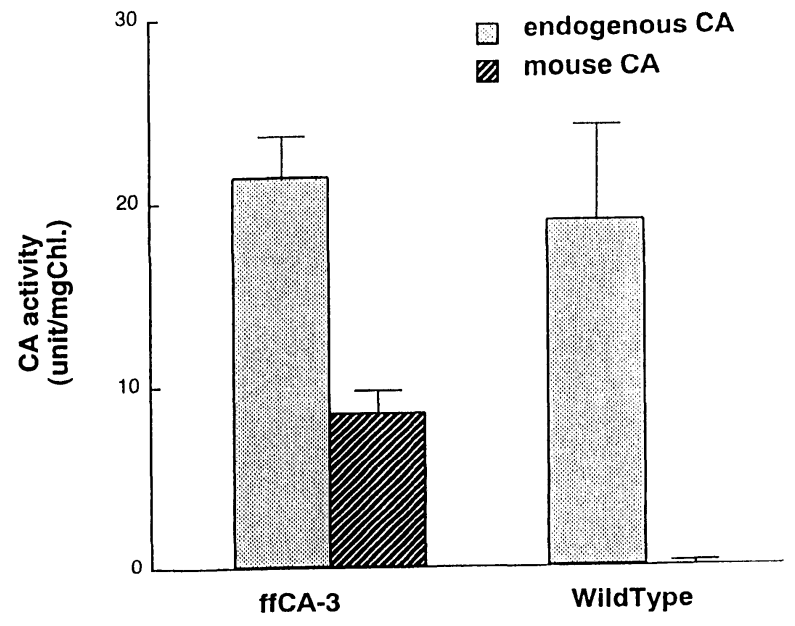

Fig. 3 Partially modified mouse CA activity in the leaf of ffCA transgenic plant (the strain $\mathrm{ffCA}^{-} 3$ ).

expression level of foreign CA compared to ffCA-3 (data not shown). From the differential CA activity measurements with and without acetazolamide (Jinushi et al., 1998), the expression levels of foreign CA activity were estimated as $8.39 \pm 1.41$ and $-0.01 \pm 0.27$ WA unit $\cdot \mathrm{mg}$ Chlorophyll ${ }^{-1}$ in the leaves of the strain ffCA- 3 and wild type, respectively. Thus, foreign CA activity occupied as high as $39.2 \%$ of total leaf CA activity in ffCA - 3 (Fig.3). Specific activity of expressed foreign CA in ffCA plants were, nearly the same level $(800 \pm 133$
WA unit / $\mathrm{mg}$ CA peptide) as those of fCA plants in the previous experiment with c.v. Xanthi $(841 \pm 58$ and $851 \pm 163$, for High CA phenotype and Low CA phenotype, respectively) (Jinushi et al., 1998).

At 8 weeks after sowing, the average expression level of foreign CA was $425.1 \pm 15.0 \mathrm{ng} \cdot \mathrm{cm}^{-2}$ in the T2 plant of ffCA-3 (Table 1). While leaf RubisCO contents in ffCA plants showed no difference from that in the wild type. The PCA rates of ffCA-3 were significantly higher than those of the wild type even at the ambient air condition $(\mathrm{Ci}=$ 250ppm) where little improvement could be seen in the fCA previously reported (Jinushi et al., 1998) (Table 2). At $250 \mathrm{ppm}$ of $\mathrm{Ci}$, the PCA rate of the transgenic plant was $14.1 \pm 0.1 \mu \mathrm{mol} \mathrm{CO}{ }_{2} \cdot \mathrm{m}^{-2} \cdot \mathrm{s}^{-1}$, which was $2.9 \%$ higher than that of the wild type. Moreover, at $100 \mathrm{ppm}$ of $\mathrm{Ci}$, the transgenic plants showed $6.7 \%$ higher PCA rate than that of the wild type. However, compared to fCA plants the percentages of the improvement of PCA rate at lower $\mathrm{CO}_{2}$ concentration did not increase drastically in ffCA plants. This might indicate the existence of a certain saturation level of cytoplasmic foreign CA activity for the improvement of PCA rate in tobacco plants. Regarding this point, it was reported that $\mathrm{Rr}$ might not be a major limiting step in the C3 PCA process (Sasaki et al., 1996). Our data showed little effects of foreign CA on the PCA rate at such a low light

Table 1. The levels of mouse CA, RubisCO and chlorophyll in the leaves of ffCA (SR - I) and fCA (Xanthi) transgenic tobacco plants.

\begin{tabular}{lccc}
\hline & \multicolumn{3}{c}{ The leaf content of } \\
& mouse CA & Rubis CO & chlorophyll \\
\cline { 2 - 4 } & $\mathrm{ng} \mathrm{cm}^{-2}$ & $\mu \mathrm{g} \mathrm{cm}^{-2}$ & $\mu \mathrm{g} \mathrm{cm}^{-2}$ \\
\hline ffCA plants (n=4) & $425.1 \pm 15.0$ & $201.9 \pm 7.7$ & $40.5 \pm 1.0$ \\
Wild type (n=5) & --- & $207.7 \pm 5.5$ & $41.3 \pm 1.1$ \\
\hline Reference data from the previous report with Xanthi & & \\
fCA plants ( $\mathrm{n}=9)$ & $277.0 \pm 8.1$ & $203.0 \pm 4.8$ & $40.4 \pm 0.6$ \\
Wild type $(\mathrm{n}=8)$ & -- & $207.8 \pm 4.1$ & $41.7 \pm 0.8$ \\
\hline
\end{tabular}

Table 2. The PCA rate in ffCA transgenic plants under high light condition of $1000 \mu \mathrm{molE}^{-2} \mathrm{~S}^{-1}$.

\begin{tabular}{lcccc}
\hline & \multicolumn{4}{c}{ PCA rate at $\mathrm{CO}_{2}$ concentrations of } \\
& $\mathrm{Ci} 300 \mathrm{ppm}$ & $\mathrm{Ci} 250 \mathrm{ppm}$ & $\mathrm{Ci} 150 \mathrm{ppm}$ & $\mathrm{Ci} 100 \mathrm{ppm}$ \\
\hline & \multicolumn{4}{c}{$\mu \mathrm{mol} \mathrm{CO} \mathrm{m}^{-2} \mathrm{~s}^{-1}$} \\
\hline ffCA plants (n=4) & $16.9 \pm 0.2^{*}$ & $14.1 \pm 0.1^{* *}$ & $8.21 \pm 0.05^{* * *}$ & $4.48 \pm 0.05^{* *}$ \\
Wild type (n=5) & $16.5 \pm 0.1$ & $13.7 \pm 0.1$ & $7.78 \pm 0.10$ & $4.20 \pm 0.08$ \\
Enhancement (ffCA/WT) x100 & $2.4 \%$ & $2.9 \%$ & $5.5 \%$ & $6.7 \%$ \\
\hline
\end{tabular}

${ }^{*}, * *$ and ${ }^{* * *}: 5,2.5$ and $1 \%$ significance, respectively. 
intensity as $500 \mu \mathrm{E} \cdot \mathrm{m}^{-2} \cdot \mathrm{s}^{-1}$ and a higher $\mathrm{CO}_{2}$ concentration such as $300 \mathrm{ppm}$, namely, $6.65 \pm 0.16$ and $6.79 \pm 0.21 \mu \mathrm{mol} \mathrm{CO}{ }_{2} \cdot \mathrm{m}^{-2} \cdot \mathrm{s}^{-1}$, ffCA plants $(n=4)$ and wild type $(n=5)$, respectively. It can be concluded that the optimal manifestation of the cytoplasmic CA introduction effect on C3 photosynthesis is only observable under light saturated and $\mathrm{CO}_{2}$ supply limiting conditions.

We also conducted the simultaneous measurement of chlorophyll fluorescence parameters. No significant differences in the calculated quantum yield of PS II ( $\Phi$ II ) was observed between ffCA transgenic plants and the wild type (data not shown). However, the $\mathrm{Rr}$ of ffCA plants was significantly lower than that of the wild type. On the other hand, we could detect no differences in stomatal resistance (Rs) and mesophyll resistance for $\mathrm{CO}_{2}$ fixation ( $\mathrm{Rx}$ ) between ffCA and wild type (Table 3). Therefore, these results indicated that the improvement of PCA rate under $\mathrm{CO}_{2}$ limiting conditions occurrs with the introduction of foreign CA activity in mesophyll cytoplasm through the reduction of $\mathrm{Rr}$. The failure to detect any significant improvement in PCA rate under lower light conditions where $\mathrm{Rr}$ was not a limiting factor in PCA (von Caemmerer and Farquhar, 1981) would support this hypothesis.

As to the growth observation under the natural light condition, the high-ffCA transgenic plants grew faster than the wild type plants. The seedlings of strain ffCA-3 showed $10 \%$ larger leaf area and 7 $\%$ heavier total dry weight than wild type plants at 7 weeks after sowing (Table 4), although there was no significant change in the low-ffCA transgenic plant, ffCA-1. In the similar experiment under low light condition with $50 \%$ shading of the natural light intensity, ffCA-3 did not show any growth

Table 3. Total resistance (Rt), stomatal resistance (Rs), mesophyll resistance for $\mathrm{CO}_{2}$ transfer(Rr) and mesophyll resistance for $\mathrm{CO}_{2}$ fization $(\mathrm{Rx})$ in $\mathrm{ffCA}$ transgenic plants under the condition of $1000 \mu \mathrm{molEm}^{-2} \mathrm{~s}^{-1}$ of light intensity and $\mathrm{Ci}=250 \mathrm{ppm}$.

\begin{tabular}{|c|c|c|c|c|}
\hline & Rt & Rs & $\mathrm{Rr}$ & $\mathrm{Rx}$ \\
\hline & \multicolumn{4}{|c|}{$\mathrm{molm}^{-2} \mathrm{~s}^{-1}$} \\
\hline ffCA plants $(n=4)$ & $20.9 \pm 0.2^{* *}$ & $3.18 \pm 0.10$ & $6.20 \pm 0.18^{* *}$ & $11.5 \pm 0.2$ \\
\hline Wild types $(n=5)$ & $21.7 \pm 0.2$ & $3.44 \pm 0.19$ & $6.81 \pm 0.17$ & $11.4 \pm 0.1$ \\
\hline
\end{tabular}

$* *: 2.5 \%$ significance.

Table 4. Leaf area, total dry weight, mouse CA and chlorophyll content in the ffCA transgenic plants under natural light condition.

\begin{tabular}{|c|c|c|c|c|c|}
\hline & \multicolumn{2}{|c|}{ Total Leaf Area } & \multirow{2}{*}{$\frac{\text { Total Dry Weight }}{\mathrm{g}}$} & \multirow{2}{*}{$\frac{\text { Leaf mouse CA }}{\mathrm{ng} \cdot \mathrm{cm}^{-2}}$} & \multirow{2}{*}{$\begin{array}{l}\text { Leaf Chl. } \\
\mu \mathrm{g} \cdot \mathrm{cm}^{-2}\end{array}$} \\
\hline & & $\mathrm{cm}^{2}$ & & & \\
\hline & 5 weeks & 7weeks & 7weeks & 7weeks & 7 weeks \\
\hline $\mathrm{ffCA}^{-3} \quad(\mathrm{n}=12)$ & $17.1 \pm 0.5$ & $532.4 \pm 15.0^{* *}$ & $1.69 \pm 0.05^{*}$ & $235.4 \pm 23.6$ & $33.2 \pm 0.4^{*}$ \\
\hline $\mathrm{ffCA}^{-1} \quad(\mathrm{n}=7)$ & $17.0 \pm 0.4$ & $486.9 \pm 18.8$ & $1.55 \pm 0.07$ & $80.3 \pm 14.3$ & $32.7 \pm 0.6$ \\
\hline Wild type $(n=10)$ & $17.1 \pm 0.4$ & $483.9 \pm 15.1$ & $1.58 \pm 0.05$ & - & $31.7 \pm 0.8$ \\
\hline
\end{tabular}

$*$ and ${ }^{* *}: 5$ and $2.5 \%$ significance, respectively.

Lable 5. Leaf area, total dry weight, mouse $\mathrm{CA}$ and chlorohpyll content in the leaf of ffCA trnasgenic plants under $50 \%$ shading natural light condition.

\begin{tabular}{|c|c|c|c|c|c|c|}
\hline & & \multicolumn{2}{|c|}{ Total Leaf Area } & \multirow{2}{*}{$\begin{array}{c}\text { Total Dry Weight } \\
\mathrm{g}\end{array}$} & \multirow{2}{*}{$\frac{\text { Leaf mouse CA }}{\mathrm{ng} \cdot \mathrm{cm}^{-2}}$} & \multirow{2}{*}{$\begin{array}{l}\text { Leaf Chl. } \\
\mu \mathrm{g} \cdot \mathrm{cm}^{-2}\end{array}$} \\
\hline & & & $m^{2}$ & & & \\
\hline & & 5weeks & 9weeks & 9weeks & 9weeks & 9weeks \\
\hline ffCA- 3 & $(n=4)$ & $10.0 \pm 0.5$ & $449.6 \pm 24.6$ & $0.97 \pm 0.08$ & $116.4 \pm 9.2$ & $27.6 \pm 0.5$ \\
\hline Wild type & $(n=7)$ & $10.3 \pm 0.4$ & $502.4 \pm 20.8$ & $1.07 \pm 0.05$ & - & $27.6 \pm 0.4$ \\
\hline
\end{tabular}


enhancement (Table 5). These findings corresponded well to those of photosynthetic analysis data and suggested the positive effects of introduced cytoplasmic CA on their growth rate under light saturated and $\mathrm{CO}_{2}$ supply limiting conditions. However, there remain alternative possible indirect reasons induced by the introduction of foreign CA activity in the cytoplasm connected to the observed growth enhancement: 1) Leaf chlorophyll and $\mathrm{Ru}-$ bisCO contents increased along with the plant size in the vegetative stage of tobacco seedlings. Hence, the transgenic plants with higher PCA activity might have relatively thick and photosynthetically active leaves at an earlier growing stage compared with the wild type plants (Table 4). 2) When transgenic plants grew faster for the reasons above, they might have expanded their leaves faster than wild type plants. Therefore, they might have higher light capturing efficiency at the whole plant level, which brought about further growth enhancement. 3) Under extremely high light conditions, stomatal closure would lead a decrease in the internal $\mathrm{CO}_{2}$ concentration of the leaf bringing about photosynthetic photoinhibition. Enhancement of leaf carbon utilization efficiency by the introduced cytoplasmic CA activity might prevent mesophyll tissues from accumulating extra-reducing power. Regarding this, the reduction of photoinhibition is one of the major target points of the recent crop breeding challenge in molecular biology.

Price et al. (1994) carried out suppression experiments of endogenous CA activity by means of antisense mRNA technology and reported that the drastic reduction of tobacco leaf endogenous CA activity to 1 or $2 \%$ of wild type made little effect on its PCA rate. Thus, it was considered that there should be too much CA activity in the mesophyll cells of $\mathrm{C} 3$ plants and any over-expression of CA activity in the mesophyll cells (in the chloroplasts) might not contribute to improvement of its PCA rate (Majeau et al., 1994). However, the results of our previous and present paper suggested that the level of CA activity in choloroplasts in $\mathrm{C} 3$ tobacco appears to be saturated in nature but the specific introduction of CA activity into the cytoplasmic area of mesophyll cells would improve PCA activity through reduction of $\mathrm{Rr}$ even with a small increase range of the introduced CA activity.

\section{Acknowledgments}

This study was partly supported by both the ADVANCE DDS Promotion Grant (91-98, ADVANCE Co. Ltd., Tokyo) and the Grant in Aid given to RI from the Ministry of Education, Culture and Science of Japan (No.07456008).

\section{References}

Broun, G., Selegny, E., Minh, C.T., Thomas, D., 1970. Facilitated transport of $\mathrm{CO}_{2}$ across a membrane bearing carbonic anhydrase. FEBS Letters, 7: 223-226.

Evans, J.R., Sharkey, T.D., Berry, J.A., Farquhar, G.D., 1986. Carbon- isotope discrimination measured concurrently with gas-exchange to investigate $\mathrm{CO}_{2}$ diffusion in leaves of higher plants. Aust. J. Plant Physiol., 13: $281-292$.

Evans, J.R., von Caemmerer, S., Setscell, B.A., Hudson, G.S., 1994. The relationship between $\mathrm{CO}_{2}$ transfer conductance and leaf anatomy in transgenic tobacco with a reduced content of RubisCO. Australian Journal Plant Physiol., 21: 475-495.

Genty, B., Briantais, J.M., Baker, N.R., 1989. The relationship between the quantum yield of photosynthetic electron transport and quenching of chlorophyll fluorescence. Biochim. Biochem. Acta, 990: 87-92.

Harley, P.C., Loreto, F., DiMarco, G., Sharkey, T.D., 1992. Theoretical considerations when estimating the mesophyll conductance to $\mathrm{CO}_{2}$ flux by analysis of the response of photosynthesis to $\mathrm{CO}_{2}$ Plant Physiol., 98: 1429- 1436.

Horsch, R.B., Fry, J.E., Hoffmann, N.L., Eichholtz, D., Rogers, S.G. and Fraley, R.T., 1985. A simple and general method for transferring genes into plants. Science, 227: 1229- 1280.

Jinushi, K., Okabe, K., Ishii, R., 1998. Photosynthetic carbon assimilation of transgenic tobacco plant expressing cytoplasmic carbonic anhydrase cDNA from mouse. Plant Biotech., 15: 235-238.

Kay, R., Chan, A., Daly, M., McPherson, J., 1987. Duplication of CaMV35S promoter sequences creates a strong enhancer for plant genes. Science, 236: 12991302.

Loreto, F., Harley, P.C., DiMarco, G., Sharkey,T.D., 1992. Estimation of mesophyll conductance to $\mathrm{CO}_{2}$ flux by three different methods. Plant Physiol., 98: 1437- 1443.

Majeau, N., Arnoldo, M. and Coleman, J.R., 1994. Modification of carbonic anhydrase activity by anti-sense and over-expression constructs in transgenic tobacco. Plant Mol. Biol., 25: 377- 385.

Mitsuhara, I., Ugaki, M., Hirochika, H., Ohshima, M., Murakami, T., Gotoh, Y., Katayose, Y., Nakamura, S., Honkura, R., Nishimiya, S., Ueno, K., Mochizuki, A., Tanimoto, H., Tsugawa, H., Otsuki, Y., Y. Ohashi, 1996. Efficient promoter cassettes for enhanced expression of foreign genes in dicotyledonous and monocotyledonous plants. Plant Cell Physiol., 37: 49-59.

Nobel, P.S., Zaragoza, L.J., Smith, W.K., 1975. Relation between mesophyll surface area, PCA rate, and illumination level during development for leaves of Plectranthus parviflorus Henckel. Plant Physiol., 55: 10671077.

Price, G.D., von Caemmerer, S., Evans, J.R., Yu, J.W., Lloyd, J., Oja, V., Kell, P., Harrison, K., Gallagher, A., Badger, M.R., 1994. Specific reduction of chloroplast carbonic anhydrase activity by anti-sense RNA in 
transgenic tobacco plants has a minor effect on photosynthetic $\mathrm{CO}_{2}$ assimilation. Planta, 193: 331-340.

Rumeau, D., Cuine, S., Fina, L., Gault, N., Nicole, M., Pertie, G., 1996. Subcellular distribution of carbonic anhydrase in Solanum tuberosum L. leave. Planta, 199: 79-88.

Sasaki, H., Samejima, M., Ishii, R., 1996. Analysis by $\delta{ }^{13} \mathrm{C}$ measurement on mechanism of cultivar difference in leaf photosynthesis of rice (Oriza sativa L.). Plant Cell Physiol., 37: 1161-1166.

Schreiber, U., 1986. Continuous recording of photochemical and non-photochamical chlorophyll fluorescence quenching with a new type of modulation fluorometer. Photosynthesis Res., 9: 261- 272.

Svab,Z., Maliga,P., 1991. Mutation proximal to the tRNA binding region of the Nicotiana plastid $16 \mathrm{~S}$ rRNA confers resistance to spectinomycin. Mol. Gen. Gene., 228: $316-319$.

Tsuzuki, M., Miyachi, S., Edwards, G.E., 1985. Localization of carbonic anhydrase in terrestrial C3 plants. Plant Cell Physiol., 26: 881-891.

Utsunomiya, E., Muto S., 1993. Carbonic anhydrase in the plasma membranes from leaves of $\mathrm{C} 3$ and $\mathrm{C} 4$ plants. Physiol. Plant., 88: 413-419.

von Caemmerer, S., Evans, J.R., 1991. Determination of the average partial pressure of $\mathrm{CO}_{2}$ in chloroplasts from leaves of several C3 plants. Aust. J. Plant Physiol., 18: 287-305.

von Caemmerer, S., Farquhar, G.D., 1981. Some relationships between the biochemistry of photosynthesis and gas exchange of leaves. Planta, 153: 376-387. 